\title{
Drug therapies in severe asthma - the era of stratified medicine 垱”
}

\author{
Authors: Kathy J Hetherington ${ }^{A}$ and Liam G Heaney ${ }^{B}$
}

\begin{abstract}
Difficult-to-treat asthma affects up to $20 \%$ of patients with asthma and is associated with significant healthcare cost. It is an umbrella term that defines a heterogeneous clinical problem including incorrect diagnosis, comorbid conditions and treatment non-adherence; when these are effectively addressed, good symptom control is frequently achieved. However, in $3-5 \%$ of adults with difficult-to-treat asthma, the problem is severe disease that is unresponsive to currently available treatments. Current treatment guidelines advise the 'stepwise' increase of corticosteroids, but it is now recognised that many aspects of asthma are not corticosteroid responsive, and that this 'one size fits all' approach does not deliver clinical benefit in many patients and can also lead to side effects. The future of management of severe asthma will involve optimisation with currently available treatments, particularly corticosteroids, including addressing non-adherence and defining an 'optimised' corticosteroid dose, allied with the use of 'add-on' target-specific novel treatments. This review examines the current status of novel treatments and research efforts to identify novel targets in the era of stratified medicines in severe asthma.
\end{abstract}

KEYWORDS: Severe asthma, stratified medicine, personalised medicine

\section{Introduction}

Up to $20 \%$ of patients with asthma do not achieve an acceptable level of control, despite being prescribed highdose anti-inflammatory and bronchodilator therapy, and are referred to as having 'difficult-to-treat asthma' or 'difficult asthma.' ${ }^{1,2}$ Difficult asthma is not a diagnosis, but is an umbrella term to describe a clinical problem that requires careful assessment. In many cases, after detailed systematic evaluation, a coexistent problem is identified (see Box 1$){ }^{3}$ either alone (misdiagnosis) or together with mild/moderate asthma, and when this is effectively managed, symptoms can be controlled.

Authors: ${ }^{A}$ research fellow, Centre for Infection and Immunity, Health Sciences Building, Belfast, UK; ${ }^{\text {B }}$ Clinical professor, Centre for Infection and Immunity, Health Sciences Building, Belfast, UK
Box 1. Coexistent conditions that can cause

persistent 'asthma-like' symptoms, from case series

of difficult asthma. Reproduced with permission. ${ }^{3}$

$>$ Dysfunctional breathlessness

$>$ Vocal cord dysfunction

> Hyperventilation with panic attacks

$>$ Obesity/physical deconditioning

$>$ Chronic obstructive pulmonary disease

Bronchiolitis obliterans

> Cardiac disease - congestive heart failure, cardiomyopathy

$>$ Pulmonary vascular disease

$>$ Bronchiectasis/cystic fibrosis

$>$ Immunodeficiency

$>$ Hypersensitivity pneumonitis

$>$ Allergic bronchopulmonary aspergillosis

$>$ Hypereosinophilic syndromes/Churg-Strauss syndrome

Acquired tracheobronchomalacia

However, within this group of patients with difficult asthma, some patients will have severe refractory asthma, where persistent symptoms and exacerbations are due to asthma that cannot be controlled with currently available treatments. Current asthma international treatment guidelines advocate a 'one size fits all' approach to treating asthma with progressive escalation of treatment, particularly corticosteroid treatment, to try to achieve asthma control. Indeed, the current definition of severe asthma mandates escalation to high-dose treatment (high-dose inhaled corticosteroids plus second controller for the previous year or systemic corticosteroids $\geq 50 \%$ of the previous year), which either maintains asthma control or fails to achieve control. ${ }^{4}$ Uncontrolled asthma is defined as persisting asthma symptoms, frequent severe exacerbations requiring at least two bursts of systemic corticosteroids (or a hospitalisation) in the previous year, or persistent airflow limitation. ${ }^{4}$ Controlled asthma that worsens on tapering of high-dose corticosteroid treatment or use of additional biological therapy also fits into the category of severe disease. It is important to stress that a diagnosis of severe refractory asthma is applied only after detailed systematic 
multidisciplinary assessment for patients with asthma in whom alternative diagnoses have been excluded, adherence with treatment has been checked, comorbidities have been treated, and trigger factors have been removed (where this is possible). With the advent of multiple new therapeutic options for severe asthma over the next few years (discussed below), it is more critical than ever to get the diagnosis and these core basic issues correct to avoid inappropriate targeting of these novel and potentially expensive therapies. The specialist commissioning of severe asthma services currently being advanced by NHS England endorses this type of approach in regional difficult asthma centres, and aims to deliver systematic assessment and specialist care in tertiary respiratory centres providing multidisciplinary diagnostic, assessment and treatment service for individuals with severe asthma. It also states that a key role is '... to act as gatekeepers for the use of bronchial thermoplasty and omalizumab, as well as other high cost novel biological agents currently in development, to prevent inappropriate use, unnecessary risk to patients and spiralling costs to the NHS' ${ }^{5}$

A clear example of where this systematic approach is critical is addressing the challenge of non-adherence with maintenance inhaled steroid treatment, which is prevalent in patients presenting with difficult asthma. ${ }^{6,7}$ It is poorly identified in routine clinical practice, leading to poor healthcare outcome and death, and can result in patients being inappropriately labelled as having severe disease, with the potential for inappropriate escalation to systemic corticosteroids or biological therapies. In patients with difficult asthma, sputum eosinophilia has been shown to be significantly higher than in patients with severe disease who are adherent with highdose inhaled steroid treatment. ${ }^{7}$ Patients with persistent airway eosinophilia have frequent exacerbations and often have peripheral blood eosinophilia, ${ }^{8,9}$ which are likely to be the core criteria for access to the novel biological therapies targeting interleukin (IL) $-5 .{ }^{10}$ Given the poor clinical outcome, inappropriate escalation to these novel therapies seems almost inevitable unless non-adherence is identified more efficiently in specialist clinical services. Recent 'biomarker-based' assessments of corticosteroid response may identify patients who should achieve good asthma control with better adherence to standard treatment. ${ }^{11}$ An advantage of this type of testing is that it can also identify 'non-intentional' non-adherence, where despite efforts to take inhalers and good prescription filling, actual adherence is poor (poor inhaler technique, poor recall of instruction, low comprehension etc). This testing is relatively simple and can be delivered using remote technologies, and will be explored as part of the Medical Research Council-funded Refractory Asthma Stratification Programme (RASP-UK). ${ }^{12}$ A key challenge remains how to manage non-adherence effectively in this patient group, ${ }^{13}$ but the starting point is identifying that non-adherence is the clinical problem in a patient who should respond well to inhaled steroid treatment.

A number of comorbidities are commonly reported in a population with severe asthma (Box 2), and management guidelines advocate the clinical management of these comorbidities. ${ }^{4}$ However, the evidence that managing these comorbidities has a major clinical impact on asthma outcome in this population is limited. For example, despite a substantial body of literature discussing the relationship between gastro-oesophageal reflux and asthma, causality has not been
Box 2. Comorbidities commonly reported in severe asthma.

Rhinosinusitis/(adults) nasal polyps

Psychological factors: personality trait, symptom perception, anxiety, depression

$>$ Vocal cord dysfunction

Obesity and physical deconditioning

Smoking/smoking-related disease

Obstructive sleep apnoea

Hyperventilation syndrome

Hormonal influences: premenstrual, menarche, menopause, thyroid disorders

Gastro-oesophageal reflux disease

Drugs including aspirin, non-steroidal anti-inflammatory drugs, $\beta$-adrenergic blockers, angiotensin-converting enzyme inhibitors

established, and although common in all severities of asthma including difficult asthma, ${ }^{14}$ the results of antacid therapy have been disappointing. ${ }^{15}$ This may be because non-acid reflux is still occurring or because the presence of gastro-oesophageal reflux has little impact on underlying asthma. Gastrooesophageal reflux can be effectively surgically treated with fundoplication and efficacy has been suggested in asthma. ${ }^{16}$ However, a 'sham-controlled' fundoplication study has never been performed and this type of study has been useful in questioning established surgical practice in other disease areas. ${ }^{17}$ Similarly, the precise link between obesity and severe asthma remains unclear; however, discrete obese phenotypes have consistently emerged in cluster analyses of cohorts of patients with severe asthma. ${ }^{8,18}$ A number of biologically plausible interactions have been suggested, including corticosteroid insensitivity, mechanical forces involved in ventilation, hormonal influences (leptin and adiponectin) and other comorbidities such as gastro-oesophageal reflux, but the benefits of weight-loss reduction programmes and bariatric surgery remain unclear. ${ }^{19,20}$ As can be seen from these examples, a challenge for the future will be to tease out association from 'cause and effect' for all of the commonly reported morbidities in severe asthma, which will allow more evidence-based targeting of interventions, including invasive surgical procedures, in this patient group.

Patients on high-dose corticosteroid therapy, particularly where it includes recurrent systemic exposure, also develop well-recognised side effects including osteoporosis, diabetes mellitus, hypertension, cataracts, psychological disturbance and cushingoid features. ${ }^{21}$ Previous studies of immunosuppressants have included small numbers of patients in randomised controlled trials and have concluded that, although there may be small steroid-sparing effects, the sideeffect profile does not justify their use. ${ }^{22-24}$ Novel therapies targeting corticosteroid-responsive biology are likely to reduce systemic corticosteroid exposure more effectively than immunosuppressants in severe asthma. Allied with a strategy to prevent inappropriate escalation of corticosteroid treatment, 
this would deliver a step change in the iatrogenic morbidity in this group. However, in one recent study with mepolizumab, a monoclonal antibody against IL-5, only a modest reduction in the maintenance dose of oral glucocorticoids was seen (mediandose prednisone in the mepolizumab group reduced from 10.0 $\mathrm{mg}$ to $3.1 \mathrm{mg}$ per day, compared with a reduction from $12.5 \mathrm{mg}$ per day to $10.0 \mathrm{mg}$ per day in the placebo arm) and few patients managed to withdraw oral steroids completely. However, there was also a $32 \%$ reduction in exacerbations in the mepolizumabtreated group, suggesting further reduction in rescue steroid exposure. It remains to be seen whether the 'steroid-sparing' effect is clinically meaningful to patients with a reduction in steroid-induced side effects; however, the side-effect profile of this agent and other biological therapies seems very favourable. It also remains to be seen whether the benefits to patients will mitigate the costs of treatment with mepolizumab.

Recent data in patients with both mild and severe asthma suggest that there is evidence of significant disease heterogeneity, ${ }^{25-29}$ and examining gene signatures in wellcharacterised cohorts of patients with severe asthma has demonstrated that between $25 \%$ and $50 \%$ of patients have a prototypic type- 2 cytokine gene signature, so-called 'T2high' disease (with elevated IL-4, -5 and -13 and airway eosinophilia, despite adherence with high-dose corticosteroid treatment). In patients with severe asthma with no evidence of T2 inflammation ('T2-low asthma'), it is likely that their corticosteroid dose has been escalated inappropriately to try to manage persistent symptoms that are not corticosteroid responsive. Given the evidence that corticosteroid responsiveness is confined to T2-high disease, ${ }^{25,30,31}$ a key challenge for the management of severe asthma in the future is to develop objective tests and validated management algorithms not only to initiate corticosteroid treatment, but also to allow clinicians to determine that additional corticosteroid treatment will not produce any further clinical response. Moving away from the currently advocated symptom-driven escalation of corticosteroid treatment will be a major component of delivering 'personalised treatment' in severe asthma in the future, and will facilitate optimisation of corticosteroid dose. It would also allow a diagnosis of severe asthma to be made without escalation of corticosteroid treatment past a point where, in many cases, there is unlikely to be any therapeutic benefit.

Patients with T2-high disease have refractory eosinophilic asthma where, despite adherence with high-dose inhaled corticosteroids, there is persistent type- 2 cytokine-driven inflammation and airway eosinophilia. Currently, these patients frequently require systemic corticosteroids to improve disease control, but the therapeutic management of this group of patients with severe asthma will be transformed over the next decade with the advent of additional novel target-specific therapies targeting the T2-cytokine axis. Omalizumab is currently available in the clinic and is a recombinant DNAderived humanised immunoglobulin (Ig) G1K monoclonal antibody that binds selectively to human IgE at the same site as the high-affinity IgE receptor, forming immune complexes with free $\operatorname{IgE} .^{32}$ This binding inhibits interaction of IgE with IgE receptors on the surface of mast cells, basophils and other cell types, preventing the release of inflammatory mediators that occurs in allergic asthma. Clinical trials have demonstrated reduced unscheduled emergency visits and hospital admissions, and current guidelines advocate the use of omalizumab as an add-on therapy in severe asthma. ${ }^{32}$

Many new biological therapies targeting T2-high disease will be available in the next five years and will generate many interesting questions, including differential efficacy between monoclonal antibodies targeting IL-5/the IL-5 receptor (IL-5R) (mepolizumab, benralizumab, reslizumab), IL-13 (lebrikizumab, tralokinumab) and IL-4R $\alpha$ (dupilumab). Other strategies targeting the $\mathrm{T} 2$ axis, including anti-CrTh2 and novel anti-IgE therapies (quilizumab, MEDI-4212, ligelizumab), will also be targeting overlapping patient groups; identifying which patients respond better to different classes of drugs may require 'head-to-head' studies. Many of these new therapies will come to market with a companion diagnostic or predictive biomarker of clinical response, which is a current limitation of omalizumab.

Bronchial thermoplasty delivers radio-frequency energy to the airways with the aim of reducing airway smooth muscle mass and hyperresponsiveness, ${ }^{33}$ although its role in the management of severe asthma remains to be established, particularly with the advent of target-specific pharmacological therapies.

A major currently unmet need in severe asthma is therapeutic management of T2-low/non-corticosteroid-responsive facets of severe asthma. It now seems clear that asthma symptoms and altered physiology are manifest in the absence of T2cytokine/eosinophilic inflammation, but it is unclear what underlying pathophysiological mechanisms drive these processes. Possibilities include a different inflammatory process or non-inflammatory structural problems such as abnormal smooth muscle contractility, aberrant epithelial signalling or airway infection. Understanding the extent and mechanism of T2-low disease in severe asthma will be a major research focus in the next decade. The role of IL-17 in asthma has generated interest, and an initial study using anti-IL-17 (brodalumab) suggested some efficacy in asthma patients with significant bronchodilator reversibility, ${ }^{34}$ but the precise role of IL-17 in severe asthma needs to be established. Preliminary data using macrolide therapy in patients with low peripheral blood eosinophil counts have also suggested exacerbation reduction, but whether this is an antibacterial or other anti-inflammatory effect remains to be clarified. ${ }^{35}$ However, optimising corticosteroid treatment and the availability of novel targetspecific treatments will provide a great opportunity to identify novel mechanisms in severe disease.

The Medical Research Council has recently funded an ambitious stratified medicines programme in severe asthma (RASP-UK). ${ }^{12}$ The primary focus of this programme is to target corticosteroid treatments more effectively, including widespread implementation of biomarker-based strategies to identify non-adherence and prevent inappropriate escalation of therapy. The programme will also examine a novel composite biomarker strategy to optimise corticosteroid therapy, which if successful will be easily transferred into routine clinical care. Future work will aim to understand why some patients and some aspects of the asthma syndrome do not respond to corticosteroids.

The greatest future challenge in severe asthma remains a 'disease-modifying' therapy. It is attractive to speculate that if we could understand why patients with a particular pattern 
of disease (T2-high/eosinophilic), which is usually responsive to low doses of inhaled corticosteroids, becomes 'relatively' corticosteroid resistant and requires high-dose (often systemic) treatment, we could target this therapeutically. This area has been the subject of study for many years, but no precise mechanism, as evidenced by a proven therapeutic, has yet emerged. In a randomised controlled withdrawal study of omalizumab (the evaluating Xolair persistency of response after long-term therapy (XPORT) study), 176 patients who had been on omalizumab for at least five years were randomised to placebo or continuation of omalizumab. ${ }^{36}$ The placebo arm had a shorter time to first exacerbation than those who continued on omalizumab; however, notably, $47.7 \%$ of placebo patients had no exacerbation in a one-year follow-up period and it is interesting to speculate that some patients have a persistent benefit; however, further analysis and studies are required to determine whether this is the case. It is also worth noting that over $25 \%$ of those randomised were not on inhaled corticosteroids at the time of randomisation and it remains unclear whether these data can be extrapolated to patients with severe asthma on omalizumab who frequently require highdose inhaled steroid treatment in addition to omalizumab to maintain disease control. However, what can be concluded is that, as a group, patients who withdraw omalizumab after longterm use do less well than those who continue treatment.

\section{Conclusion}

Severe asthma represents a significant unmet medical need. Novel therapeutics, targeting a particular severe asthma phenotype (T2-high/refractory eosinophilic), are arriving in the clinic and will substantially increase management options for this group. Precise clinical assessment, with a particular focus on adherence with inhaled steroid treatment, is critical to ensure that these therapies are used in the correct patient group. The arrival of these therapies will allow the research focus to shift towards understanding T2-low mechanisms in severe asthma.

\section{References}

1 Global Initiative for Asthma. Global strategy for asthma management and prevention. Available online at www.ginasthma.org/local/ uploads/files/GINA_Report_2014_Aug12.pdf [Accessed 19 March 2015].

2 Hekking PP, Wener RR, Amelink M et al. The prevalence of severe refractory asthma. J Allergy Clin Immunol 2015;135:896-902.

3 Heaney LG, Robinson DS. Severe asthma treatment: need for characterising patients. Lancet 2005;365:974-6.

4 Chung KF, Wenzel SE, Brozek JL et al. International ERS/ATS guidelines on definition, evaluation and treatment of severe asthma. Eur Respir J 2014;43:343-73.

5 NHS England. A14/S/1b - 2013/14 NHS standard contract for respiratory: severe asthma (adult). Available online at www.england. nhs.uk/wp-content/uploads/2013/06/a14-respiratory-sev-asthma. pdf [Accessed 19 March 2015].

6 Gamble J, Stevenson M, McClean E, Heaney LG. The prevalence of nonadherence in difficult asthma. Am J Respir Crit Care Med 2009;180:817-22.

7 Murphy AC, Proeschal A, Brightling CE et al. The relationship between clinical outcomes and medication adherence in difficultto-control asthma. Thorax 2012;67:751-3.
8 Haldar P, Pavord ID, Shaw DE et al. Cluster analysis and clinical asthma phenotypes. Am J Respir Crit Care Med 2008;178:218-24.

9 Fowler SJ, Tavernier G, Niven R. High blood eosinophil counts predict sputum eosinophilia in patients with severe asthma. J Allergy Clin Immunol 2015;135:822-4.

10 Ortega HG, Liu MC, Pavord ID et al. Mepolizumab treatment in patients with severe eosinophilic asthma. $N$ Engl J Med 2014;371:1198-207.

11 McNicholl DM, Stevenson M, McGarvey LP, Heaney LG. The utility of fractional exhaled nitric oxide suppression in the identification of nonadherence in difficult asthma. Am J Respir Crit Care Med 2012;186:1102-8.

12 Medical Research Council Refractory Asthma Stratification Programme in the United Kingdom (RASP-UK). Moving away from the 'one size fits all' approach to treatment in severe asthma. Available online at www.rasp.org.uk/ [Accessed 19 March 2015].

13 Nieuwlaat R, Wilczynski N, Navarro T et al. Interventions for enhancing medication adherence. Cochrane Database Syst Rev 2014;11:1-730.

14 Leggett JJ, Johnston BT, Mills M et al. Prevalence of gastroesophageal reflux in difficult asthma: relationship to asthma outcome. Chest 2005;127:1227-31.

15 Gibson PG, Henry RL, Coughlan JL. Gastro-oesophageal reflux treatment for asthma in adults and children. Cochrane Database Syst Rev 2003;2:1-33.

16 Rothenberg SS, Bratton D. The effects of laparoscopic Nissen fundoplication to enhance pulmonary function in the treatment of a patient with severe asthma and gastroesophageal reflux disease. J Allergy Clin Immunol 2008;121:1069-70.

17 Sihvonen R, Paavola M, Malmivaara A et al. Arthroscopic partial meniscectomy versus sham surgery for a degenerative meniscal tear. N Engl J Med 2013;369:2515-24.

18 Moore WC, Meyers DA, Wenzel SE et al. Identification of asthma phenotypes using cluster analysis in the Severe Asthma Research Program. Am J Respir Crit Care Med 2010;181:315-23.

19 Gibson PG. Obesity and asthma. Ann Am Thorac Soc 2013;10 Suppl:S138-42.

20 Dias-Júnior SA, Reis M, de Carvalho-Pinto RM et al. Effects of weight loss on asthma control in obese patients with severe asthma. Eur Respir J 2014;43:1368-77.

21 Sweeney J, Brightling CE, Menzies-Gow AN et al. Regular oral corticosteroid use increases the risk of steroid related morbidity in refractory asthma compared to frequent rescue courses - data from the British Thoracic Society (BTS) Difficult Asthma Registry. Am J Respir Crit Care Med 2014;189:A2426.

22 Evans DJ, Cullinan P, Geddes DM. Cyclosporin as an oral corticosteroid sparing agent in stable asthma. Cochrane Database Syst Rev 2001;2:1-17.

23 Davies H, Olson L, Gibson P. Methotrexate as a steroid sparing agent for asthma in adults. Cochrane Database Syst Rev 2000;2:157.

24 Bel EH, Wenzel SE, Thompson PJ et al. Oral glucocorticoid-sparing effect of mepolizumab in eosinophilic asthma. N Engl J Med 2014;371:1189-97.

25 Woodruff PG, Modreck B, Choy DF et al. T-helper type 2-driven inflammation defines major subphenotypes of asthma. Am J Respir Crit Care Med 2009;180:388-95.

26 Jia G, Erickson RW, Choy DF et al. Periostin is a systemic biomarker of eosinophilic airway inflammation in asthmatic patients. J Allergy Clin Immunol 2012;130:647-54.

27 Butler CA, McQuaid S, Taggart CC et al. Glucocorticoid receptor $\beta$ and histone deacetylase 1 and 2 expression in the airways of severe asthma. Thorax 2012;67:392-8.

28 Shikotra A, Choy DF, Ohri CM et al. Increased expression of immunoreactive thymic stromal lymphopoietin in patients with severe asthma. J Allergy Clin Immunol 2012;129:104-11. 
29 Choy D, Shikotra A, Siddiqui S et al. Mutually exclusive Th2 and Th17 bronchial gene expression signatures are associated with eosinophilia in asthma. Eur Respir J 2014;44 Suppl 58;P3868.

30 McGrath KW, Icitovic N, Boushey HA et al. A large subgroup of mild-to-moderate asthma is persistently noneosinophilic. Am J Respir Crit Care Med 2012;185:612-19.

31 Pavord ID, Brightling CE, Woltmann G, Wardlaw AJ. Non-eosinophilic corticosteroid unresponsive asthma. Lancet 1999;353;2213-14.

32 Rodrigo GJ, Neffen H, Castro-Rodriguez JA. Efficacy and safety of subcutaneous omalizumab vs placebo as add-on therapy to corticosteroids for children and adults with asthma: a systematic review. Chest 2011;139:28-35.

33 Castro M, Rubin AS, Laviolette $\mathrm{M}$ et al. Effectiveness and safety of bronchial thermoplasty in the treatment of severe asthma: a multicenter, randomized, double-blind, sham-controlled clinical trial. Am J Respir Crit Care Med 2010;181:116-24.
34 Busse WW, Holgate S, Kerwin E et al. Randomized, double-blind, placebo-controlled study of brodalumab, a human anti-IL-17 receptor monoclonal antibody, in moderate to severe asthma. Am J Respir Crit Care Med 2013;188:1294-302.

35 Brusselle GG, Joos G. Is there a role for macrolides in severe asthma? Curr Opin Pulm Med 2014;20:95-102.

36 Busse WW, Trzaskoma B, Omachi TA et al. Evaluating Xolair persistency of response after long-term therapy (XPORT). Am J Respir Crit Care Med 2014;189:A6576.

Address for correspondence: Prof L Heaney, Centre for Infection and Immunity, Queen's University of Belfast, Belfast City Hospital, Lisburn Road, Belfast BT9 7AB, UK. Email: I.heaney@qub.ac.uk

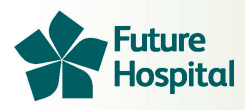

\section{Everyone has a story to tell Tell us yours...}

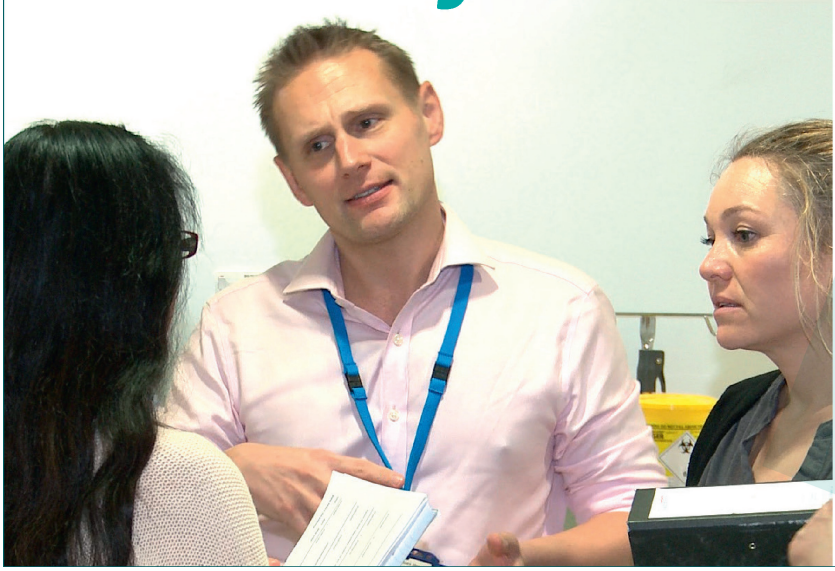

The Future Hospital Programme (FHP) aims to develop and implement the RCP's vision for the future of medical care in hospital and community settings. We are keen to showcase examples of best and innovative practice to improve patient care, and would very much like to hear more about your work.

We are asking people to share their stories to create, grow and connect a future hospital network. These stories of best and innovative practice and patient care may be featured on the RCP website as exemplars of FHP principles and recommendations in action.

To share your story

Visit: www.rcplondon.ac.uk/FH-tell-us-your-story

For further information

Visit: www.rcplondon.ac.uk/FHP

or contact

futurehospital@rcplondon.ac.uk 